\title{
Yod
}

Revue des études hébraïques et juives

19 | 2014

Aharon Appelfeld, cinquante ans d'écriture

\section{Les secrets de la mémoire et l'art de la transmission}

L'histoire et l'histoire de l'histoire

Secrets of Memory and Art of Transmission

על סודות הזיכרון ואמנות המסירה

\section{Masha Itzhaki}

\section{OpenEdition}

\section{Journals}

Édition électronique

URL : https://journals.openedition.org/yod/2014

DOI : 10.4000/yod.2014

ISSN : 2261-0200

Éditeur

INALCO

\section{Édition imprimée}

Date de publication : 30 mai 2014

ISBN : 978-2-85831-214-6

ISSN : 0338-9316

Référence électronique

Masha Itzhaki, "Les secrets de la mémoire et l'art de la transmission », Yod [En ligne], 19 | 2014, mis en ligne le 26 janvier 2016, consulté le 08 juillet 2021. URL : http://journals.openedition.org/yod/2014 DOI : https://doi.org/10.4000/yod.2014

Ce document a été généré automatiquement le 8 juillet 2021.

\section{c) (i) (3)}

Yod est mis à disposition selon les termes de la Licence Creative Commons Attribution - Pas d'Utilisation Commerciale 4.0 International. 


\section{Les secrets de la mémoire et l'art de la transmission}

L'histoire et l'histoire de l'histoire

Secrets of Memory and Art of Transmission

על סודות הזיכרון ואמנות המסירה

\section{Masha Itzhaki}

Le jeu de cache-cache

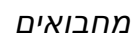

בחצר אחורית בעולם

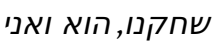

כסיתי עיני, התחבאואי ואני

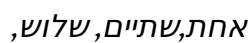

לא מלפני,לא מאחים, שלורי,

לא בתוכי. - לא מלפי,

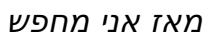

כל כך הרבה שנים.

אז מה אם כר אני לא מוצא נשים אותך.

צא כבר, צא, אני אני

אתה רואה שנכנעתי.

Dans l'arrière-cour du monde

On jouait, lui et moi,

Je fermais les yeux, il se cachait :

Un, deux, trois,

Pas devant moi, pas derrière,

Pas en moi.

Depuis, je cherche

Depuis tant et tant d'années.

Et si je ne te trouve pas...

Sors, allez sors,

Tu vois, je me rends ${ }^{1}$. 


\section{Les secrets de la mémoire}

1 Le sous-titre de cet article, "L'histoire et l'histoire de l'histoire », exprime d'une certaine façon la double trame qui constitue l'ensemble de la narration d'Aharon Appelfeld, celle qui englobe une mémoire, personnelle et collective à la fois, et celle qui cherche perpétuellement la meilleure façon de la transmettre. Le quoi et le comment, l'histoire et l'histoire de l'histoire.

2 Dans son ouvrage le plus autobiographique, Histoire d'une vie ${ }^{2}$, Appelfeld évoque la nature floue de la mémoire humaine. En fait, l'auteur lui-même, dans son préambule, considère son ouvrage comme l'histoire conflictuelle de sa propre mémoire à travers laquelle on pourrait dessiner les étapes principales d'un processus psychologique caractéristique des survivants de la Shoah et en particulier de ceux parmi eux qui effectuent un travail de transmission. Tout d'abord, l'oubli du pire par la création de «trous noirs ", résultat direct d'un refoulement, acte inconscient de défense par lequel le moi rejette des épisodes douloureux de son passé afin de reprendre la vie, une étape que l'auteur dénomme תרדמת השכח, le sommeil de l'oubli³. Puis, des accès soudains, provoqués inconsciemment pour des raisons indéterminées, à des souvenirs occasionnels qui se bloquent de nouveau par la suite ; cela constitue une lutte à laquelle participent toutes les composantes de l'âme. Enfin, la quête du sens et de la cohérence dans une tentative de relier ces fragments isolés de la mémoire selon telle ou telle logique pour rendre la transmission possible ${ }^{4}$.

3 Pour surmonter le mutisme et trouver le chemin vers une écriture qui ne soit pas falsificatrice et parvienne malgré tout à exprimer l'inconcevable et le silence, il fallait accomplir un long parcours, déchiffrer les secrets de la mémoire de l'enfance et les traduire en un mode d'emploi qui garde l'essentiel en évitant le compulsif. Et tout cela, dans une langue nouvelle, acquise, l'hébreu.

4 À plusieurs reprises, Appelfeld soutient que le seul véhicule de la mémoire capable d'empêcher la falsification est de caractère corporel et non pas intellectuel. Noms, dates et lieux pourraient facilement tomber dans l'oubli, pourtant c'est le corps qui se souvient du passé à travers une sensation de froid, la pluie, une odeur, une voix. Survivre à la guerre est, avant tout, de nature physique : cela se traduit par une quête de pain, d'eau, d'un abri pour les nuits glaciales, d'un peu de chaleur. Ce ne sont donc pas les mots qui restent gravés dans la mémoire, mais les sensations. Ainsi, c'est à travers les fragments où les déclencheurs physiques mènent aux souvenirs de sensations que le point de vue de l'enfant se forme. L'auteur adulte avoue avoir emmagasiné uniquement la mémoire de l'enfance, celle qui ignore les détails de nature historique pour conserver seulement des images ponctuelles, des sentiments forts, mais non verbalisés, typiques plutôt du dynamisme obscur qui constitue les rêves. C'est pourquoi, dans le préambule d'Histoire d'une vie, l'auteur met son lecteur en garde, comme s'il lui disait entre les lignes: «Je ferai de mon mieux pour vous raconter l'histoire, mais mon mieux est limité, car je suis piégé par moi-même, je suis obligé de me protéger. » En effet, les souvenirs concernant directement la guerre se composent plutôt d'éclats de mémoire et produisent un élément majeur d'instabilité et de surprise, ils n'obéissent à aucune cohérence intellectuelle, ni à un calendrier attendu. Ils sont complètement imprévisibles : "Parfois passe un mois sans même une vision de ces jours. Ce n'est qu'une pause, évidemment. Il suffit tout simplement d'un vieil objet laissé sur le bord de la route pour faire surgir des profondeurs des centaines de pieds 
qui piétinent dans un long convoi où il n'y a personne pour relever celui qui s'effondre ${ }^{5}$."

D'autres déclencheurs de la mémoire sont les couleurs, surtout le vert et le noir. Les années dans la forêt sont marquées par le vert, une sensation particulière qui revient en marchant pieds nus sur le gazon; le noir, par contre, fait peur : même adulte en plein Jérusalem ; le noir c'est l'obscurité, l'absence d'issue, une longue nuit de guerre. À cela on peut ajouter les odeurs, celles de la paille pourrie et de la boue, ainsi que les sons, un cri d'oiseau, de l'eau qui coule et surtout le silence. Il fallait garder le silence partout et à tout prix : parler constituait une menace, un signe d'identité à haut risque ; le silence au contraire aiguisait l'attention. Il fallait écouter pour détecter les dangers. "Aujourd'hui encore, nous dit-il, je fais quelques pas et je m'arrête, pour écouter. Parler m'est difficile, ce qui n'est pas étonnant : durant la guerre on ne parlait pas ${ }^{6}$."

Peu à peu Appelfeld parvient à décrire le cercle vicieux de son écriture du désastre : un mot par hasard, un mot venu du passé, peut en effet évoquer une image, une sensation, un souvenir lointain et refoulé et pour verbaliser cette image il faut trouver d'autres mots, des mots propres, peu de mots, sinon, on risque de tomber dans le piège du faux, de l'exagéré, du pathos.

\section{L'art de la transmission}

7 Cette recherche perpétuelle de la mémoire nourrit une écriture qui fonctionne selon un mécanisme extrêmement élaboré: elle est constituée de matériaux empruntés à l'expérience personnelle de l'auteur, de ceux qu'il tire de l'histoire d'autres personnes et enfin, de ceux qui sont fictifs, mais réels et jouent le jeu littéraire de la vraisemblance.

8 Nous, lecteurs de la narration portant sur le désastre, avons l'habitude de réfléchir sur la question de la vérité et du parallèle entre la vie de l'auteur telle qu'on peut la reconstituer et la vie de l'auteur telle qu'il la raconte. On applique sans même le savoir la logique de l'autobiographie qui identifie personnage principal, narrateur et auteur. Or, aucun roman d'Appelfeld ne peut être considéré comme autobiographique dans le sens classique du terme.

9 En fait, l'ensemble de son écriture est une fiction construite sur le réel, qu'il soit emprunté à son vécu à lui, aux histoires des autres ou bien à ce qui est vraisemblable. Outre la narration du désastre, il fait revivre un passé plus lointain, celui des Juifs d'avant-guerre, bourgeois et assimilés d'une part et hassidim de l'autre. L'auteur crée des narrateurs variés, hommes, femmes, enfants, qui font le «va-et-vient » entre ses sources d'inspiration, entre le véridique et l'imaginaire, le dit et le non-dit, le mémorisable et le vraisemblable qui ensemble construisent ce qu'il appelle « cent ans d'histoire juive ".

10 Le besoin de s'exprimer, de raconter l'Histoire existe et devient de plus en plus urgent, exigeant, harcelant. « La peur ancienne que l'histoire de notre vie, la mienne et celle de mon ami T., ainsi que celle de nos parents et de nos grands-parents seraient enfouies à jamais, qu'il n'en resterait plus rien, cette peur me hante parfois la nuit ", dit-il dans

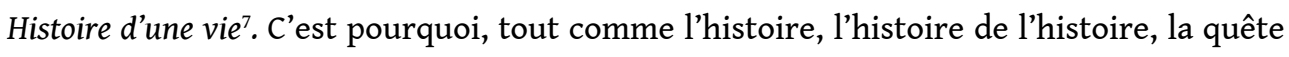
de la transmission à travers l'écriture, occupe une place à part entière dans les romans d'Appelfeld. 
11 En effet, la bataille pour les mots, les verbes, les phrases et les espaces, pour la parole d'une part et le silence de l'autre, est primordiale non seulement dans l'histoire de la vie de l'auteur, mais aussi dans le parcours de nombreux personnages de l'univers narratif d'Appelfeld; elle est aussi présente que la recherche perpétuelle de la mémoire. On peut par exemple évoquer Kuti, le jeune garçon bègue de מסע אל החורף («Voyage vers l'hiver»), qui trouve un remède à son handicap en copiant des versets hébraïques de la prière juive; Bruno du roman והזעם עוד לא נדם ("Et la fureur ne s'est pas encore tue ») qui, après cinquante ans d'une vie tourmentée, arrive à travers son écriture à une certaine tranquillité d'esprit, c'est un écrivain unique en son genre, un manchot qui puise sa création au plus profond de son malheur inconsolable ; Ernest du roman פתאום אהבה ("L'amour soudain»), retraité malade vivant à Jérusalem dont l'écriture reste inachevée durant des années et qui, à la fin de sa vie, mourant, atteint enfin sa mémoire d'enfance, la seule source d'inspiration capable de rendre cette mission possible. Ils ont tous la même histoire: tout d'abord, il faut survivre, puis trouver la mémoire et enfin transmettre l'histoire.

Ce triple parcours est au cœur du roman האיש שלא פסק לישוןtraduit en français en 2011 sous le titre «Le garçon qui voulait dormir ». Erwin, le narrateurprotagoniste, est en effet un jeune homme, sauvé des horreurs de la guerre par des rescapés adultes qui l'ont littéralement porté, car il était plongé dans un profond sommeil, tout au long des chemins de l'Europe d'après-guerre jusqu'à Naples. L'histoire de son rétablissement, d'abord à Naples puis en Palestine, est surtout l'histoire de son réveil progressif et paradoxalement - comme nous l'avons vu à plusieurs reprises - le retour à la vie doit passer par une blessure physique handicapante dans une bataille au kibboutz d'une part et par l'apprentissage de l'écriture en hébreu de l'autre. Le handicap physique est toujours une source de pouvoir, moral ou mystique, au-delà du concret. C'est le cas de Bruno Broumhart dans La fureur et c'est aussi le cas de ce jeune homme aux jambes cassées dans Le garçon qui voulait dormir qui cherche à se faire soigner afin de retrouver la mobilité physique. Son handicap lui donne un pouvoir, le pouvoir de renaître ${ }^{8}$. Erwin, qui devient en Palestine Aharon, passe une longue période à l'hôpital afin de pouvoir retrouver son autonomie. Parallèlement à ce processus médical, il se libère peu à peu de ses crises de sommeil profond et plus encore, il passe son temps à copier des textes hébreux (surtout des versets bibliques et des passages tirés de l'œuvre de S. Y. Agnon) pour enfin maîtriser la nouvelle écriture. Ce roman, tout comme Voyage vers l'hiver ou La Pologne une terre verte, raconte le récit d'une quête identitaire dont le trajet est double. La recherche de soi à travers l'axe du sommeil constitue la clé pour aboutir à l'écriture et retrouver ainsi le «chez-soi » tant recherché.

Pourquoi le sommeil ? Pour les survivants qui portent Erwin lors du périple européen, il s'agit de récupération physique, comme si l'adolescent était en train de se fortifier de l'intérieur en dormant. Leur regard correspond en fait à la définition la plus courante du sommeil: un état qui permet de récupérer pour être capable d'attaquer une nouvelle journée dans de bonnes conditions physiques. En vérité, ce sommeil profond d'Erwin n'a rien de corporel ou de physique. Bien au contraire, il sert comme un outil pour remplir doucement un vide total, le vide qui résulte de l'oubli, le vide d'un passé disparu, évaporé. C'est un sommeil métaphysique où par un vagabondage nocturne, le passé, venant visiter l'homme endormi, lui reconstruit doucement sa mémoire disparue. C'est le mécanisme du rêve qui sert ici de véhicule pour retrouver le passé, 
car, selon Appelfeld, un homme sans passé est un homme sans avenir et le passé est constitué de souvenirs.

De nombreux passages décrivant le monde rêvé illustrent le roman de la première à la dernière page. À leur lecture, on a l'impression que ce monde, tiré du passé et perdu à jamais, est en fait plus réel que le présent et c'est ainsi que le processus psychologique s'achève: autant la vie en dormant semble réelle - les rencontres rêvées avec les parents disparus sont lucides - autant le jeune homme se remplit d'énergie et de force pour faire face à son nouvel univers. La coupure radicale qui résulte du refoulement profond présente un vrai danger pour l'avenir. «La non-acceptation du vécu devient vite un ennemi », dit déjà l'auteur dans Le retrait, un essai publié en 1979.

Et l'écriture? Elle aussi est une arme contre l'oubli, le seul moyen de capter les rencontres nocturnes et les images rêvées et de les sauvegarder à jamais, la seule possibilité de reconstruire un passé vivant de "chez soi ", de l'immortaliser, tout en sachant qu'il n'existe plus. La conquête de l'écriture est une sorte de rédemption: "Quand tout devient noir et s'écroule sous le poids de tes défaites, une porte magique s'ouvre et t'invite à entrer dans ta première maison, ta maison éternelle qui t'attend depuis toujours comme tu l'avais laissée. La mienne est là depuis que j'avais six ans ${ }^{9}$. ”

L'intermédiaire du rêve construit donc par le biais du sommeil la maison magique d'Erwin, oubliée par la rupture radicale de la guerre. L'écriture d'Aharon la sauvegarde. Mais paradoxalement, Aharon décrit en hébreu la maison de son enfance, celle de ses parents et de ses grands-parents située dans un monde lointain, vécu en allemand et en yiddish et perdu à jamais. La conquête de cette langue, appartenant à un univers nouveau, est longue et douloureuse, tout comme le rétablissement physique. Le parcours n'est pas encore achevé, mais il dégage un esprit optimiste : le jeune narrateur arrive à englober son passé dans son présent avec un regard d'espoir sur l'avenir. D'ailleurs, il n'est pas le seul. Le roman décrit un ensemble de jeunes rescapés, les amis d'Erwin du camp de transit et du kibboutz. Il y a parmi eux ceux qui arrivent à reconstruire au moins une partie de leur passé (par la musique, par le dessin ou même tout simplement en gardant leurs anciens noms) pour avancer dans l'avenir et ceux qui échouent, ceux qui vivent donc et ceux qui se donnent la mort.

Il est clair que ce récit comporte certains traits autobiographiques. Le seul autre protagoniste dénommé Erwin dans l'ensemble de l'œuvre d'Appelfeld est le jeune garçon du roman מכרה הקרח («La mine de glace »). Notre Erwin, devenu Aharon comme son créateur, est-il le même? Le camp de transit, le séjour au kibboutz, le combat pour acquérir l'hébreu, le contact presque mystique avec sa mère morte, tous ces éléments sont tirés sans doute de l'histoire vraie de l'écrivain. De plus, les premiers pas d'écriture du jeune protagoniste ${ }^{10}$ sont tirés, presque mot pour mot, des premiers textes de l'auteur, soit de ses poèmes d'adolescent, soit d'une de ses premières nouvelles לאט ("Lentement », dans 'Ashan) ${ }^{11}$. Cependant, nous le savons déjà, les écrits d'Appelfeld sont empruntés à des fragments de souvenirs personnels, à des séquences isolées tirées des récits des autres ou bien à ce qui lui semble vraisemblable. De toute façon, il me semble qu'enfin, à l'âge avancé de presque quatre-vingts ans, l'adolescent qui est toujours caché en lui, a trouvé sa véritable maison dans sa langue, l'hébreu, qui abrite à la fois son passé et son présent, son enfance et sa vie d'adulte, un vrai « chez soi ».

18 Or, l'œuvre d'Appelfeld nécessite toujours une double, sinon triple, lecture. En effet, l'analyse du narrateur romanesque Erwin-Aharon permet une compréhension plus 
subtile du parcours de son créateur. C'est ainsi qu'on travaille les traits autobiographiques et c'est ainsi que fonctionne la narration de soi. Mais ce roman avance aussi une vision globale portant un point de vue novateur sur la question de l'identité israélienne.

À travers l'histoire du jeune survivant, l'auteur propose un discours alternatif à l'ancien discours sioniste du «nouveau Juif ». Il crée un héros qui retrouve son identité juive perdue et sa culture européenne diasporique, justement durant la guerre d'Indépendance d'Israël, période où l'éthos sioniste classique est au pouvoir et dicte le comportement et les pensées de la population juive de Palestine dans son ensemble. C'est un personnage pour lequel cette retrouvaille est vitale pour qu'il puisse surmonter sa faiblesse d'après-guerre, même si elle contredit complètement l'opinion publique israélienne en cette période de crise. C'est une démarche identitaire unique en son genre, considérée par son créateur (Erwin? Aharon? Appelfeld ?) comme la seule clé capable d'ouvrir les portes vers une véritable intégration et une acceptation honnête de l'autre dans un pays dont la raison d'être est basée sur l'immigration. Pour construire un nouveau "chez soi », il est nécessaire de sauvegarder celui de la diaspora ; pour accepter une nouvelle identité, il est nécessaire d'y inclure l'ancienne. Et pour ce faire, il est indispensable de faire réapparaitre la mémoire cachée, celle de l'histoire, et de la transmettre par l'histoire de l'histoire.

\section{BIBLIOGRAPHIE}

APPELfELD, Aharon (1997) מכרה הקרח (« La mine de glace »), Jérusalem, Keter.

APPELFELD, Aharon (1999) סיפור חיים, Jérusalem, Keter. Traduction française : Histoire d'une vie, Paris, Éditions de l'Olivier, 2004.

APPELFELD, Aharon (2000) מסע אל החורף ("Voyage vers l'hiver »), Jérusalem, Keter.

APPELfELD, Aharon (2001) פתאום אהבה, Jérusalem, Keter. Traduction française : L'amour soudain, Paris, Éditions de l'Olivier, 2004.

APPELfELD, Aharon (2008) והזעם עוד לא נדם Or Or Yehuda, Kinneret, Zmora-Bitan. Traduction

française : Et la fureur ne s'est pas encore tue, Paris, Éditions de l'olivier, 2009.

APPelfELD, Aharon (2010) האיש שלא פסק לישון, Or Yehuda, Kinneret, Zmora-Bitan. Traduction française : Le garçon qui voulait dormir, Paris, Éditions de l'olivier, 2011.

DUdAI, Rina (2013) “From Excess to Origin”, Yod nº 19.

FRIEDLANDER, Saul (1978) Quand vient le souvenir, Paris, Seuil.

ITZHAKI, Masha (2011) Aharon Appelfeld : le réel et l'imaginaire, Paris, L'Harmattan.

Oron, Yair (1993) זהות יהודית ישראלית («Identité juive israélienne »), Tel Aviv, Sifriat Poalim.

SEGEV, Tom (1993) Le septième million, Paris, Liana Levi. 


\section{NOTES}

1. Dan Pagis, Kol ha-shirim, Jérusalem 1971, p. 177. Traduit par l'auteur de l'article.

2. Jיפור חיים, Jérusalem, Keter, 1999. Traduction française : Paris, Éditions de l'Olivier, 2004. Les références dans cet article renvoient à l'édition hébraïque.

3. Pour le refoulement, voir entre autres Yair Oron (1993), p. 89 ainsi que Tom Segev (1993), en particulier le chapitre « Un État, les derniers Juifs».

4. Saul Friedlander (1978) construit le sens de ses souvenirs par la technique de la double narration, celle du passé transposée parallèlement à celle du présent ; vers la fin de son livre il évoque la problématique du sens : "Ce récit approche de son terme - - - et les mots de Gustav Meyrink surgissent à nouveau: 'Quand vient la connaissance, le souvenir vient aussi, progressivement...' Séquence inversée cependant : quand vient le souvenir, la connaissance vient aussi progressivement... » (p. 166-167).

5. Histoire d'une vie, p. 84.

6. Ibid. p. 95.

7. P. 165.

8. Voir à ce sujet l'article de Yitzhak Ben-Mordechai dans ce numéro.

9. Le garçon qui voulait dormir, p. 219.

10. Voir p. 188 et 210.

11. Voir à ce propos l'article de Rina Dudai.

\section{RÉSUMÉS}

Pour trouver le chemin vers une écriture qui ne soit pas falsificatrice et parvienne malgré tout à exprimer l'inconcevable et le silence, Aharon Appelfeld a dû accomplir un long parcours, déchiffrer les secrets de la mémoire de l'enfance et les traduire en un mode d'emploi qui garde l'essentiel en évitant le compulsif. Et tout cela, dans une langue nouvelle, acquise, l'hébreu. Cet article présente une étude sur le concept même de la mémoire dans l'univers de l'écrivain et analyse sa façon de transmettre l'inconcevable. Son point de départ est Histoire d'une vie (1999) pour conclure avec une analyse du roman Le garçon qui voulait dormir (2010).

In order to find his very own style, which avoids forgery and pathos, and expresses the inexpressible, Aharon Appelfeld accomplished a very long and painful course. He had to decode the secrets of childish memories and to transform them into an essential and purified narration. Thus, in modern Hebrew, totally new and acquired language. This paper is dealing with the very special concept of memory in Appelfeld's writings beginning with Story of Life (1999) and concluding with The Man Who Never Stopped Sleeping (2010).

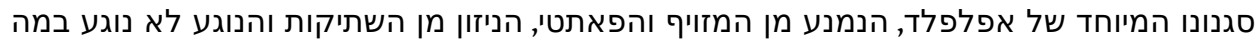

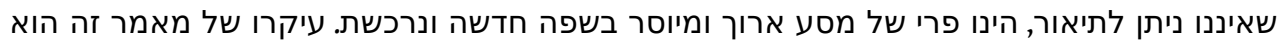

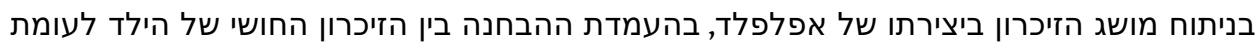

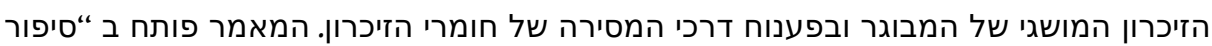

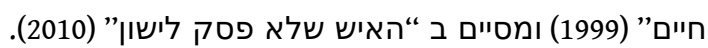


INDEX

\section{מילות מפתח}

אפלפלד, זיכרון, מסירה, אוטוביוגרפיה, סיפור חיים, האיש שלא פסק לישון:

Keywords : Appelfeld Aharon (1932-), memory, transmission, autobiography, Story of Life, The Man Who Never Stopped Sleeping, literature

Mots-clés : Appelfeld Aharon (1932-), mémoire, transmission, autobiographie, Histoire d'une vie, Le garçon qui voulait dormir

Thèmes : littérature

Index chronologique : Shoah

\section{AUTEUR}

MASHA ITZHAKI

INALCO 